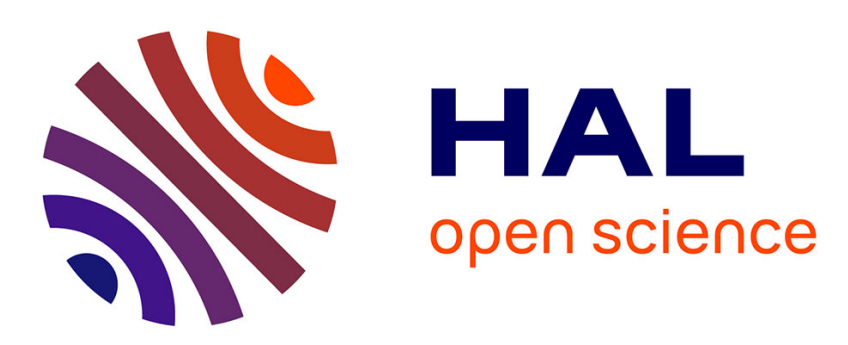

\title{
Conformational flexibility of nitroxide biradicals determined by X-band PELDOR experiments
}

Dominik Margraf, Bela E. Bode, Andriy Marko, Olav Schiemann, Thomas F.

Prisner

\section{- To cite this version:}

Dominik Margraf, Bela E. Bode, Andriy Marko, Olav Schiemann, Thomas F. Prisner. Conformational flexibility of nitroxide biradicals determined by X-band PELDOR experiments. Molecular Physics, 2008, 105 (15-16), pp.2153-2160. 10.1080/00268970701724982 . hal-00513149

\author{
HAL Id: hal-00513149 \\ https://hal.science/hal-00513149
}

Submitted on 1 Sep 2010

HAL is a multi-disciplinary open access archive for the deposit and dissemination of scientific research documents, whether they are published or not. The documents may come from teaching and research institutions in France or abroad, or from public or private research centers.
L'archive ouverte pluridisciplinaire HAL, est destinée au dépôt et à la diffusion de documents scientifiques de niveau recherche, publiés ou non, émanant des établissements d'enseignement et de recherche français ou étrangers, des laboratoires publics ou privés. 


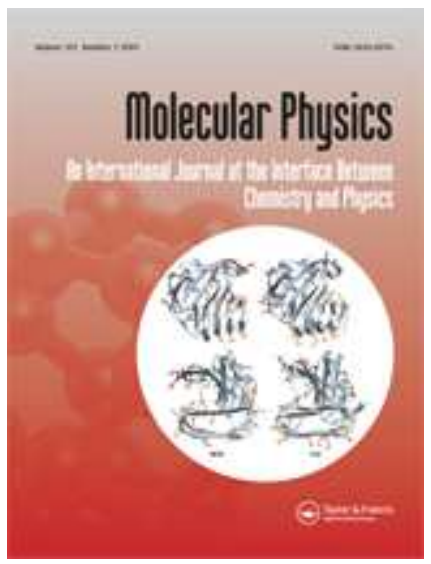

\section{Conformational flexibility of nitroxide biradicals determined by $\mathrm{X}$-band PELDOR experiments}

\begin{tabular}{|c|c|}
\hline Journal: & Molecular Physics \\
\hline Manuscript ID: & TMPH-2007-0180.R1 \\
\hline Manuscript Type: & Invited Article \\
\hline $\begin{array}{r}\text { Date Submitted by the } \\
\text { Author: }\end{array}$ & 22-Aug-2007 \\
\hline Complete List of Authors: & $\begin{array}{l}\text { Margraf, Dominik; J.W. Goethe-University } \\
\text { Bode, Bela; J.W. Goethe-University } \\
\text { Marko, Andriy; J.W. Goethe-University } \\
\text { Schiemann, Olav; J.W. Goethe-University } \\
\text { Prisner, Thomas; J.W. Goethe-University }\end{array}$ \\
\hline Keywords: & PELDOR, EPR, Simulation, Nitroxide \\
\hline
\end{tabular}

\section{(s) ScholaroNE \\ Manuscript Central}




\title{
Conformational flexibility of nitroxide biradicals
}

\section{determined by X-band PELDOR experiments}

\author{
D. Margraf†, B.E. Bode†, A. Marko†, O. Schiemann†, T.F. Prisner†*
}

\begin{abstract}
$\dagger$ Institute of Physical and Theoretical Chemistry and Center of Biomolecular Magnetic Resonance, J.W. Goethe-University Frankfurt am Main, Max-von-Laue-Str. 7, 60438 Germany

*Corresponding author. Email: prisner@prisner.de
\end{abstract}

\begin{abstract}
PELDOR (pulsed electron-electron double resonance) experiments have been performed at Xband $(9 \mathrm{GHz})$ frequencies on a linear and a bent nitroxide biradical. All PELDOR time traces were recorded with the pump frequency $\nu_{\mathrm{B}}$ set at the center of the nitroxide spectra to achieve maximum pumping efficiency, while the probe frequency $v_{\mathrm{A}}$ was stepped between a frequency offset $\Delta v_{\mathrm{AB}}=v_{\mathrm{A}}-v_{\mathrm{B}}$ of +40 to $+80 \mathrm{MHz}$. The modulation frequencies and the damping of the oscillations change as a function $\Delta v_{\mathrm{AB}}$, whereas the modulation depth $\lambda$ for our investigated systems was only very slightly altered. This can be explained by the selection of different orientations of nitroxide radicals with respect to the external magnetic field as a function of frequency offset. Quantitative simulations of the PELDOR time traces could be achieved for both molecules and for all offset frequencies using a simple geometric model, described by a free rotation of the nitroxide radical around its acetylene bond and a single bending mode of the interconnecting molecular bridge. The results show that the distribution function for the relative orientations of the nitroxides with respect to each other and with respect to the dipolar vector $R$ deviates from a random distribution and thus have to be taken into account to quantitatively simulate the PELDOR traces. Vice versa, a quantitative simulation of PELDOR time traces with variable offset frequencies allows determining the conformational freedom of such molecules.
\end{abstract}




\section{Introduction}

PELDOR [1] is a powerful tool to measure distances between paramagnetic centres in the nanometer range [2]. The method has been widely applied to measure distances in spin labeled macromolecules such as polymers [3] and biomolecules [4]. The method uses the magnetic dipole-dipole interaction to probe the distance in analogy to FRET spectroscopy, where the electric dipole-dipole interaction is utilised. In both methods not only the distance, but also the orientation of the two dipoles with respect to the interconnecting distance vector $R$ between the two radical centres affects the coupling strength. Nitroxides, which are commonly used as spin labels, typically have a large rotational and conformational freedom. Therefore, in most studies the relative orientation has been considered as random, leading to the well known Pake pattern distribution of dipolar coupling strengths. In such cases the most prominent coupling strength corresponds to an orientation where the $R$-vector is perpendicular to the external magnetic field. The peak resulting from the perpendicular orientation of the $R$ vector with respect to the external magnetic field is usually chosen in order to determine the distance (deleted: $R$ ) between the two radicals. Observation of the full Pake pattern by a 4pulse PELDOR sequence [5] (Figure 1) enables to disentangle the orientation dependent dipolar coupling $D\left(\theta_{\text {dip }}\right)$ from the isotropic exchange coupling $J$ which additionally may occur for distances smaller than $2 \mathrm{~nm}$ [6]. [insert figure 1 about here]

In cases (deleted: comma) where the orientation of the radicals to the interconnecting vector $R$ is fixed, as for example for natural paramagnetic cofactors in proteins, a different situation arises if specific orientations of the radicals can be selected by the resonant microwave pulses. Here, the orientation of the radicals with respect to the $R$ vector enters into the analysis. The observed coupling strength and the modulation depth $\lambda$ as well as the attenuation of the dipolar oscillations will depend on the specific choice of the probe and the pump frequencies [2]. Especially at high magnetic field values, where the $g$-tensor anisotropy of the radicals is usually resolved, such orientation selectivity can be readily achieved [7] as has been demonstrated for two tyrosyl radicals in a ribonucleotide reductase dimer at G-band frequencies $(180 \mathrm{GHz} / 6.5 \mathrm{~T})$. In this case a strong orientation dependence of the experimentally observed dipolar oscillation frequency could be observed permitting to additionally obtain the relative orientation of the tyrosyl radicals with respect to the $R$ vector [8].

Such orientation selection and angular correlation effects have already been observed in nitroxide biradicals with polyphenyleneethynylene linkers at X-band $(9 \mathrm{GHz} / 0.3 \mathrm{~T})$, S-band $(3 \mathrm{GHz} / 0.1 \mathrm{~T})[6,9]$ and $\mathrm{W}$-band $(95 \mathrm{GHz} / 3.4 \mathrm{~T})$ [10] frequencies.

In this paper we show (deleted: comma) that these orientation effects can be quantified by $\Delta v_{\mathrm{AB}}$-dependent PELDOR measurements at X-band frequencies. A quantitative numerical simulation of the PELDOR time traces recorded as a function of $\Delta v_{\mathrm{AB}}$ can be used to derive distinct information on the conformational flexibility of such molecules.

\section{Theory}

The magnetic dipole-dipole interaction between the magnetic moments $\vec{\mu}_{A}$ and $\vec{\mu}_{B}$ of two paramagnetic centres leads to an interaction energy $\mathrm{E}$ of:

$E=\frac{\vec{\mu}_{A} \cdot \vec{\mu}_{B}}{R^{3}}-\frac{3\left(\vec{\mu}_{A} \cdot \vec{R}\right)\left(\vec{\mu}_{B} \cdot \vec{R}\right)}{R^{5}}$

where $\mathrm{R}$ is the distance between $\mathrm{A}$ and $\mathrm{B}$. In the secular approximation the dipolar Hamiltonian $H_{\text {dip }}$ can be written in the following way: 
$H_{\text {dip }}=\frac{g_{\mathrm{A}} g_{\mathrm{B}} \beta_{\mathrm{e}}^{2}}{R^{3}} S_{\mathrm{z}}^{\mathrm{A}} \quad S_{\mathrm{z}}^{\mathrm{B}}\left(1-3 \cos ^{2} \theta_{\text {dip }}\right)$

(2)

with $g_{\mathrm{A}}$ and $g_{\mathrm{B}}$ being the $g$-values of the paramagnetic molecules $\mathrm{A}$ and $\mathrm{B}, \beta_{\mathrm{e}}$ the Bohr magneton, $R$ the distance between the two molecules and $\theta_{\text {dip }}$ the angle between the external magnetic field $B_{0}$ and the $R$ vector. The dipolar splitting of the EPR transitions is therefore given by:

$\omega_{\text {dip }}=\frac{D_{\text {dip }}}{R^{3}}\left(1-3 \cos ^{2} \theta_{\text {dip }}\right)$

where the splitting constant $D_{\text {dip }}$ is $2 \pi \cdot 52 \mathrm{MHz} \cdot \mathrm{nm}^{3}$ for nitroxide radicals. The $R$ vectors in a macroscopically disordered frozen solution sample are randomly oriented with respect to the external magnetic field. As can be seen from eq. (3), $\omega_{\text {dip }}$ varies from $-2 \cdot D_{\text {dip }} / R^{3}$ to + (deleted: 1) $D_{\text {dip }} / R^{3}$. If all molecules are excited, the amplitude of this dipolar frequency distribution is described by the classical Pake pattern [11]. The edge-to-edge distance of the Pake pattern corresponds to the dipolar splitting for molecules with an angle of $\theta_{\text {dip }}=0^{\circ}$ between the $R$ vector and the external magnetic field $B_{0}$, the peak-to-peak distance to the dipolar splitting for molecules with $\theta_{\text {dip }}=90^{\circ}$. These two peaks are the most prominent observable features in Fourier-transformed 4-pulse PELDOR time traces [12].

The echo intensity $V(t)$ of a 4-pulse PELDOR sequence for a fixed orientation of the magnetic field $B_{0}$ in the A spin molecular axis system (described by polar angles $\varphi$ and $\phi$ ) is given by:

$V\left(t, v_{\mathrm{A}}, v_{\mathrm{B}}, \varphi, \phi\right)=V_{0}\left(v_{\mathrm{A}}, \varphi, \phi\right) \cdot\left(1-\lambda\left(v_{\mathrm{B}}, \Omega, \varphi, \phi\right) \cdot\left[1-\cos \left(\omega_{\text {dip }}(\varphi, \phi) \cdot t\right)\right]\right)$

where $V_{0}$ describes the A spin echo intensity for $t=0, \Omega$ is the set of Euler angles, describing the orientation of spin B in the axis system of spin A (Figure 2) and $\lambda$ is the efficiency of inversion of the dipolar coupled spin B by the pump pulse. For disordered powder samples the observed echo intensity is obtained by integration over all magnetic field orientations [insert figure 2 about here]:

$V\left(t, v_{\mathrm{A}}, v_{\mathrm{B}}\right)=\iint V\left(t, v_{\mathrm{A}}, v_{\mathrm{B}}, \varphi, \phi\right) \sin (\phi) d \phi d \varphi$

The relative orientation between spin $\mathrm{A}$ and $\mathrm{B}$ as well as with respect to the vector $R$ will all be random regarding flexible nitroxides in a biradical. In such cases the integration over all $\Omega$ values will effectively average the magnetic field orientation dependence of the modulation depth parameter $\lambda$ [13]. In such cases the integration over different magnetic field orientations of equation (5) can be readily converted into an integration over all dipolar angles:

$$
V\left(t, v_{\mathrm{A}}, v_{\mathrm{B}}\right)=V_{0}\left(v_{\mathrm{A}}\right) \cdot\left(1-\lambda\left(v_{\mathrm{B}}\right) \cdot\left[1-\int_{0}^{\pi / 2} \cos \left(\frac{D_{\mathrm{dip}}}{R^{3}}\left(1-3 \cos ^{2} \theta_{\text {dip }}\right) t\right) \sin \theta_{\text {dip }} d \theta_{\text {dip }}\right]\right)
$$

For more rigid biradicals this simplification is not valid. The pump efficiency, described by the modulation depth parameter $\lambda$ will depend on the mutual orientation of the two radicals $A$ and $\mathrm{B}$, described by the Euler angles $\Omega$. Additionally, the orientation selectivity of the excitation of spin A as a function of $v_{\mathrm{A}}$, described by $V_{0}\left(v_{\mathrm{A}}, \varphi, \phi\right)$, will lead to a distribution function $P\left(v_{\mathrm{A}}, \theta_{\text {dip }}\right)$ of dipolar angles which differs from the $\sin \left(\theta_{\text {dip }}\right)$ distribution of a Pake pattern. The echo signal intensity for a given pump and probe frequency can in such general cases be described by $[2,13]$ :

$$
V\left(t, v_{\mathrm{A}}, v_{\mathrm{B}}\right)=\int_{0}^{\pi / 2} P\left(\Delta v_{\mathrm{AB}}, \theta_{\mathrm{dip}}\right) \cdot\left[1-\cos \left(\omega_{\mathrm{dip}} \cdot t\right)\right] d \theta_{\mathrm{dip}}
$$

Thus, the observed dipolar frequency spectrum cannot be converted directly to a distance $R$, if the geometry of the biradical, and therefore the function $P\left(v_{\mathrm{A}}, \theta_{\mathrm{dip}}\right)$ is unknown. To unravel 
orientation and distance information in such cases, the excitation and pump frequencies have to be varied to excite and pump differently oriented sub-ensembles of the powder sample.

(deleted: whereas) For flexible biradicals, with a statistical distribution of orientations, the time domain PELDOR traces, as described by equation (6), can easily be simulated by Tikhonov regularization methods [14]. However, this is more demanding for conformationally restricted biradicals, because the integral kernel function of the integral in equation (7) is more complicated. We have therefore chosen a different strategy to simulate the experimental PELDOR time traces: A conformational ensemble of biradicals is created and their calculated PELDOR time traces are compared with experiments. The conformational ensemble contains a number $N$ (typically 1000 to 10000) of different conformers, each characterised by a distance vector $R$, with polar angles $(\psi, \eta)$ in the axis system of spin A and Euler angles $\Omega$ describing the mutual orientation of molecule B with respect to molecule $A$. The input data for the structure of the conformers can be generated either by molecular dynamics (MD)-studies or by a simple geometrical model of the biradical. For each of these conformers the resonance positions of molecules $\mathrm{A}$ and $\mathrm{B}$ are calculated for all orientations of the magnetic field vector $B_{0}$ in the molecular axis frame of spin A, taking anisotropic nitrogen hyperfine coupling and the anisotropy of the g-tensor into account:

$$
\begin{aligned}
& v_{\mathrm{res}}^{\mathrm{A}}=\beta_{\mathrm{e}} \cdot B_{0} \cdot g_{\text {eff }}^{\mathrm{A}}+m_{\mathrm{I}} \cdot A_{\mathrm{eff}}^{\mathrm{A}} \\
& g_{\mathrm{eff}}^{\mathrm{A}}=\sqrt{\left(g_{\mathrm{xx}} \cdot \cos \varphi \cdot \sin \phi\right)^{2}+\left(g_{\mathrm{yy}} \cdot \sin \varphi \cdot \sin \phi\right)^{2}+\left(g_{\mathrm{zz}} \cdot \cos \phi\right)^{2}} \\
& A_{\mathrm{eff}}^{\mathrm{A}}=\sqrt{\left(A_{\mathrm{xx}} \cdot \cos \varphi \cdot \sin \phi\right)^{2}+\left(A_{\mathrm{yy}} \cdot \sin \varphi \cdot \sin \phi\right)^{2}+\left(A_{\mathrm{zz}} \cdot \cos \phi\right)^{2}}
\end{aligned}
$$

$A_{\text {eff }}$ and $g_{\text {eff }}$ are the effective hyperfine couplings and $g$-values for the specific magnetic field orientation, $m_{\mathrm{I}}$ is the nuclear spin value $\left(-1,0,+1\right.$ for $\left.{ }^{14} \mathrm{~N}\right)$. Hyperfine and $g$-tensor axis are considered as collinear to the molecular axis system for our simulations. The resonance frequencies of spin B can be calculated after describing the hyperfine and $g$-tensor of spin B in the coordinate system of spin A, as given by the transformation:

$$
\begin{aligned}
& G_{\mathrm{B}}^{\mathrm{A}}=D(\Omega) G_{\mathrm{B}}^{\mathrm{B}} D^{-1}(\Omega) \\
& A_{\mathrm{B}}^{\mathrm{A}}=D(\Omega) A_{\mathrm{B}}^{\mathrm{B}} D^{-1}(\Omega)
\end{aligned}
$$

where $D(\Omega)$ is the respective rotation matrix. Additionally, an inhomogeneous linewidth of $0.6 \mathrm{mT}$ has been taken into account by a Gaussian distribution to calculate the final resonance frequencies for spin A and spin B respectively.

Using these calculated resonance frequencies the excitation efficiency of pump and probe pulses for spin A and B can easily be determined. For a microwave pulse with a Rabi oscillation frequency $\omega_{1}=\gamma B_{1}$ and a resonance frequency $\Delta \omega_{\mathrm{r}}=2 \pi\left(v_{\mathrm{mw}}-v_{\mathrm{res}}\right)$, the inversion efficiency is given by:

$$
I_{\mathrm{ex}}\left(\Delta \omega_{\mathrm{r}}, \varphi, \phi\right)=\left(\frac{1}{2}-\frac{\Delta \omega_{\mathrm{r}}^{2}+\omega_{1}^{2} \cdot \cos \left(\frac{\pi \sqrt{\Delta \omega_{\mathrm{r}}^{2}+\omega_{1}^{2}}}{\omega_{1}}\right)}{2\left(\Delta \omega_{\mathrm{r}}^{2}+\omega_{1}^{2}\right)}\right)
$$

For a large sample size the inhomogeneous field strength $B_{1}$ distribution over the resonator height can be described by a half sine-wave. Integration of equation (10) over the resonator length leads to an excitation profile which can be approximated by:

$$
I_{\text {ex }}\left(\Delta \omega_{\mathrm{r}}, \varphi, \phi\right)=\frac{\omega_{1}^{2}}{\omega_{1}^{2}+4 \cdot \Delta \omega_{\mathrm{r}}^{2}}
$$

This allows to calculate the excitation functions of spin $\mathrm{A}, V_{0}\left(v_{\mathrm{A}}, \varphi, \phi\right)$, and of spin $\mathrm{B}$, $\lambda\left(v_{\mathrm{B}}, \Omega, \varphi, \phi\right)$, and thereafter the dipolar distribution function $P\left(v_{\mathrm{A}}, \theta_{\mathrm{dip}}\right)$ for each conformer. 
The pump pulse frequency $\nu_{\mathrm{B}}$ is fixed to the centre of the nitroxide spectrum in our experiments and only the detection frequency $v_{\mathrm{A}}$ is varied. The final PELDOR signal for a given frequency offset $\Delta v_{\mathrm{AB}}$ is a sum over all magnetic field orientations equally distributed on a sphere (typically 20000) and over all conformers. All simulations are performed with a home-written MATLAB ${ }^{\circledR}$ program. A simulation of an ensemble of 1000 conformers, each of them with 20000 orientations with respect to the magnetic field takes about 3 minutes of computing time on a PC.

\section{Samples and Experimental Conditions}

Biradicals 1 and 2 (Figure 3) were synthesized according to literature [15] [insert figure 3 about here]. Samples were prepared as $50 \mu \mathrm{M}$ solutions in toluene and degassed via several freeze-thaw cycles prior to storage in liquid nitrogen. PELDOR spectra were recorded on a Bruker ELEXSYS E580 pulsed X-band EPR spectrometer with a standard flex line probehead housing a dielectric ring resonator (MD5 W1) equipped with a continuous flow helium cryostat (CF935) and temperature control system (ITC 502) both from Oxford instruments. The second microwave frequency was coupled into the microwave bridge by a commercially available setup (E580-400U) from Bruker. All pulses were amplified via a pulsed traveling wave tube (TWT) amplifier (117X) from Applied Systems Engineering. Over-coupling of the resonator led to a quality factor $Q$ of about 100. 4-pulse PELDOR experiments were performed with the pulse sequence $\pi / 2\left(v_{\mathrm{A}}\right)-\tau_{1}-\pi\left(v_{\mathrm{A}}\right)-\left(\tau_{1}+t\right)-\pi\left(v_{\mathrm{B}}\right)-\left(\tau_{2}-t\right)-\pi\left(v_{\mathrm{A}}\right)-\tau_{2}$-echo. The excitation bandwidths of pump and probe pulses have to be chosen small enough to avoid spectral overlap. On the other hand the excitation width of the inversion pump pulse should be as large as possible to achieve a deep modulation depth $\lambda$, given by the fractions of spins $\mathrm{B}$ inverted by the pump pulse. Therefore the pump pulse $\left(v_{\mathrm{B}}\right)$ was set to $12 \mathrm{~ns}$ at the resonance frequency of the resonator and applied to the maximum of the nitroxide spectrum. The pulse amplitude was then optimized to maximum inversion of a Hahn-echo on the pump frequency $v_{\mathrm{B}}$. For the simulations a pulse lengths of $16 \mathrm{~ns}$ had to be chosen to obtain the observed modulation depth $\lambda$. The difference might result from non-rectangular pulse shapes, the experimental reproducibility of $\lambda$ or adjustment of optimum pulse lengths. Detection pulses $\left(v_{\mathrm{A}}\right)$ were set to $32 \mathrm{~ns}$ and applied at frequency offsets $\Delta v_{\mathrm{AB}}=v_{\mathrm{A}}-v_{\mathrm{B}}$ from +40 to $+80 \mathrm{MHz}$ in $10 \mathrm{MHz}$ steps with constant $v_{\mathrm{B}}$. The pulse amplitudes were chosen to optimize the refocused echo and the $\pi / 2$-pulse was phase-cycled to eliminate receiver offsets. All spectra were recorded at $40 \mathrm{~K}$ with an experiment repetition time of $4 \mathrm{~ms}$, a video amplifier bandwidth of $25 \mathrm{MHz}$ and an amplifier gain of $60 \mathrm{~dB}$. $\tau_{1}$ was set to $136 \mathrm{~ns}$ and $\tau_{2}$ to $4000 \mathrm{~ns}$. Suppression of proton modulation was achieved by addition of 8 spectra of variable $\tau_{1}$ with $\Delta \tau_{1}$ of $8 \mathrm{~ns}$ [16]. Usually 720 scans were accumulated with 332 data points and time increments $\Delta t$ of $12 \mathrm{~ns}$ giving an approximate measurement time of 2 hours. Intermolecular contributions to the time domain signal were removed by division by a mono-exponential decay. The resulting spectra were normalized to $t=0$.

\section{Results}

Figure 4 shows the experimental PELDOR time traces for $\mathbf{1}$ and $\mathbf{2}$ as a function of the probe frequency offset $\Delta v_{\mathrm{AB}}$ ranging from $40-80 \mathrm{MHz}$ [insert figure 4 about here]. The pumping frequency $\nu_{\mathrm{B}}$ is for all experiments kept at the centre of the nitroxide spectra, as shown in the inset of Figure 4. As can be seen the oscillation frequencies vary as a function of $\Delta v_{\mathrm{AB}}$. This indicates the presence of angular correlation effects for $\mathbf{1}$ and 2. For the $80 \mathrm{MHz}$ offset a high orientation selectivity for the observer spin A is obtained with our chosen pulse lengths; only nitroxide molecules with the plane normal almost parallel to the external magnetic field are observed, whereas for an offset of $40 \mathrm{MHz}$ the orientation distribution of the excited 
nitroxides is broader. This is illustrated in Figure 5: The contribution to the signal is encoded in colour for each orientation of the external magnetic field with respect to the nitroxide molecular axis system [insert figure 5 about here]. Pumping in the centre of the nitroxide spectra with a $12 \mathrm{~ns} \pi$-pulse length excites all molecular orientations. Only nitroxide molecules with the plane normal parallel to the external magnetic field and in the ${ }^{14} \mathrm{~N}$ nuclear $m_{\mathrm{I}}= \pm 1$ spin states (with a large hyperfine coupling) are not excited. For the linear biradical 1, where the molecular z-axis of the two nitroxides is perpendicular to the $R$ vector (considering a rigid molecule), this orientation selection leads to simple predictions for the observable dipolar frequencies: The parallel frequency component should only appear for small frequency offsets $\Delta v_{\mathrm{AB}}$, whereas at $80 \mathrm{MHz}$, with high orientation selection, only the perpendicular component of the Pake pattern should be observable. This tendency is experimentally observed, as shown in Figure 4. On the other hand the modulation depth $\lambda$ does not change as a function of $\Delta v_{\mathrm{AB}}$ for $\mathbf{1}$ and $\mathbf{2}$. Also for the bent biradical $\mathbf{2}$ a change of oscillation frequencies is observed as a function of $\Delta v_{\mathrm{AB}}$ (Figure $4 \mathrm{~b}$ ). The effect is less pronounced, compared to $\mathbf{1}$, because for $\mathbf{2}$ a much faster damping of the oscillations occurs.

\section{Discussion}

The simple geometric model which we applied to simulate the conformational distribution of the biradicals is shown in Figure 3. The 5-ring nitroxide molecules are allowed to rotate freely around their acetylene bond axis. This leads to a random orientation of the nitroxide $\mathrm{x}$-axis $(\mathrm{N}-\mathrm{O})$ on a cone with an angle of $22^{\circ}$ with respect to the linear acetylene linker. The nitroxide $\mathrm{z}$-axis (out of plane) is perpendicular to the linear linker but otherwise randomly oriented. The conformational flexibility of the interconnecting molecular bridge is modelled by a simple bending motion, as depicted in Figure 3, with a Gaussian half width angle $\delta \phi$. The highest orientation selection and symmetry is achieved for molecule 1 with a frequency offset of 80 MHz. Under these conditions only nitroxide radicals with the normal parallel to the external magnetic field contribute to the signal. The bending angle $\delta \phi$ was optimized on the basis of this experimental data set. The best result was obtained with a bending angle of $\delta \phi=40^{\circ}$. With the same conformational distribution the frequencies, modulation depth and attenuation of the PELDOR traces obtained with other frequency offsets could also be well reproduced, as shown in Figure 6 for $\Delta v_{\mathrm{AB}}=80 \mathrm{MHz}$ and $40 \mathrm{MHz}$ [insert figure 6 about here]. Especially the damping and modulation depth can be compared much better with a simulation of the time domain data instead of often used frequency domain data. The attenuation of the oscillations is strongly influenced by the bending angle $\delta \phi$. A quantitative reproduction of the experimentally observed attenuation of the oscillations can only be achieved taking into account this relatively strong bending. The large conformational freedom of the molecular bridge is in qualitative agreement with unpublished MD predictions and published MDstudies [10], [17]. The MD and the simple conformational model both lead to a slightly shorter average distance than the predicted minimum energy structure and therefore a faster oscillation period, in agreement with the experimental data. Nevertheless, even with this fairly large flexibility of the bridge, orientation correlations exist, evident from the changes in oscillation frequencies for the smaller offset frequency of $40 \mathrm{MHz}$. Another factor that could alter the shape of the PELDOR traces with varying frequency offset is the spectral overlap between pump and probe pulses. The most prominent effect is that the short pump pulse also interacts with A spins excited by the probe pulse echo sequence. These A spins will not refocus at the echo position anymore and therefore do not contribute to the PELDOR signal. Indeed switching on the pump pulse leads to an experimentally observed reduction in echo intensity. Simulation of this interference effect for $\Delta v_{\mathrm{AB}}=40 \mathrm{MHz}$ also showed a reduction in echo intensity for the studied conformer ensemble, but no change in the PELDOR time trace shape within the experimental accuracy. This effect will be smaller for larger offset values, 
indicating that it does not contribute to the offset dependent change in oscillation frequencies for the chosen experimental conditions.

Thus, the observed change in oscillation frequency as a function of $\Delta v_{\mathrm{AB}}$ can be attributed to a different selection of dipolar angles in both experiments. Figure 7 depicts the dipolar angle distribution function $P\left(\Delta v_{\mathrm{AB}}, \theta_{\mathrm{dip}}\right)$ for 1 extracted from the simulations for $\Delta v_{\mathrm{AB}}=40 \mathrm{MHz}$ and $80 \mathrm{MHz}$ [insert figure 7 about here]. Both distributions deviate strongly from the $\sin \theta_{\text {dip }}-$ distribution expected for random orientations of the nitroxides with respect to the bridge. Whereas the distribution for $\Delta v_{\mathrm{AB}}=80 \mathrm{MHz}$ is mainly located around $\theta_{\mathrm{dip}}=90^{\circ}$ also smaller dipolar angles contribute to the simulations with $40 \mathrm{MHz}$ frequency offset. Especially values close to $\theta_{\text {dip }}=0^{\circ}$ lead to the additional faster oscillations seen in this time trace.

For biradical 2, with an angle of $60^{\circ}$ in the bridge, the same conformational model, with a free rotation of the nitroxides around the acetylene linker bond and a single bending distribution $\delta \phi$, was used to simulate the PELDOR time traces. We could reproduce the experimental PELDOR time traces for all offset frequencies well with a smaller mobility of the bridge of $\delta \phi=20^{\circ}$. Simulations and experimental data are summarised in Figure 6 for $80 \mathrm{MHz}$ and 40 $\mathrm{MHz}$ frequency offsets. The much faster damping of the oscillations is a consequence of the angle in the bridge. The bending motion causes a much more pronounced effect on the distance in this case, leading to a broader distance distribution and therefore faster damping of the oscillations. This might also explain the slightly smaller angle $\delta \phi$ for this molecule: In our simple model, where the conformational freedom of the bridge is approximated by only a single bending motion, the distance variation is obviously underestimated for the linear biradical 1.

In both cases we demonstrated that X-band PELDOR time traces are very sensitive to the conformational freedom of the biradical, which in both cases cannot be described accurately by a random orientation of the nitroxide radicals with respect to the molecular bridge. Both molecules can be reasonably well simulated by applying simple geometric models.

\section{Conclusion}

We were able to show on a set of two biradicals that the $\Delta v_{\mathrm{AB}}$-dependence of the oscillation frequencies arises from orientation correlations between the nitroxides and the distance vector $R$. Even with large bending motions of the bridge such effects may remain and hamper the analysis of the distance $R$ between the two unpaired electrons. If the experiment is performed only with one fixed offset frequency and analysed by assuming a random orientation distribution, a wrong $R$ distribution could result. This can be avoided by taking PELDOR time traces with different pump and probe frequencies. Summing all these time traces may lead to an averaging of such orientation correlations, as has been shown by Godt et al. [9]. On the other side a quantitative analysis of the frequency offset dependence will allow to gather a detailed picture of the conformational distribution of the molecule. An analogous situation might also arise in biological applications where steric constrains restrict the mobility of the spin labels. By a systematic variation of the detection frequency $v_{\mathrm{A}}$ such effects can easily be detected and taken into account. This yields additional information on the mutual orientation of the two nitroxide radicals, which might be interesting in structural studies.

Together with PELDOR experiments at different magnetic field values and under different freezing conditions such experiments will generate benchmark data for optimization of MD force field parameters and potentials for such molecules, as will be shown in an upcoming publication. 
Figure 1: 4-pulse PELDOR sequence used for these measurements. The variable time $t$ of the pump pulse is zero at the first Hahn-echo position.

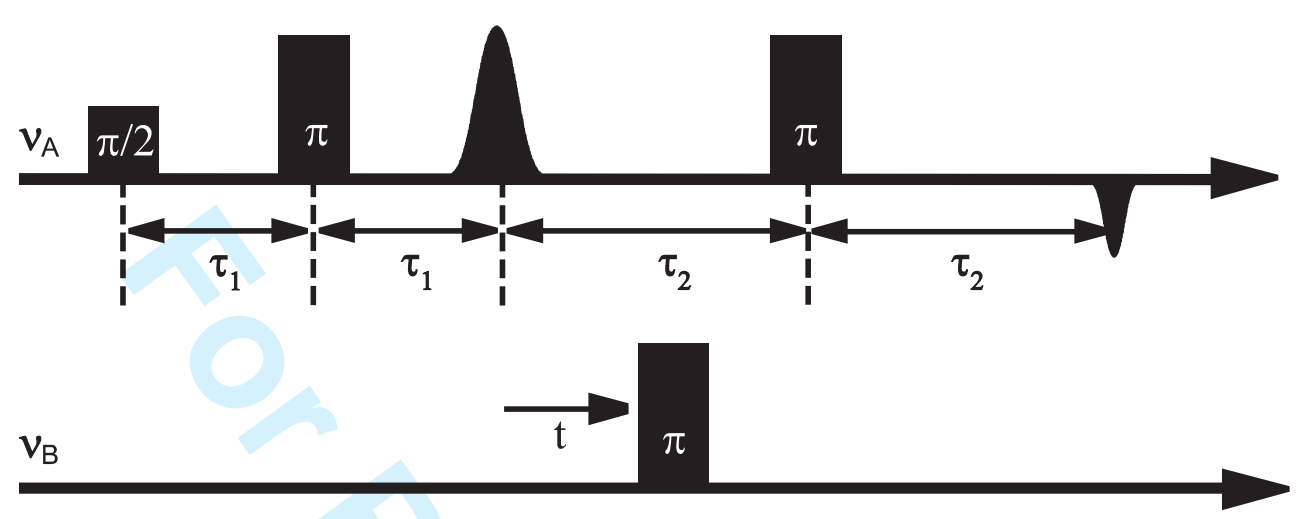

Figure 2: Definition of the axis system of the discussed biradicals $\mathbf{1}$ and 2.

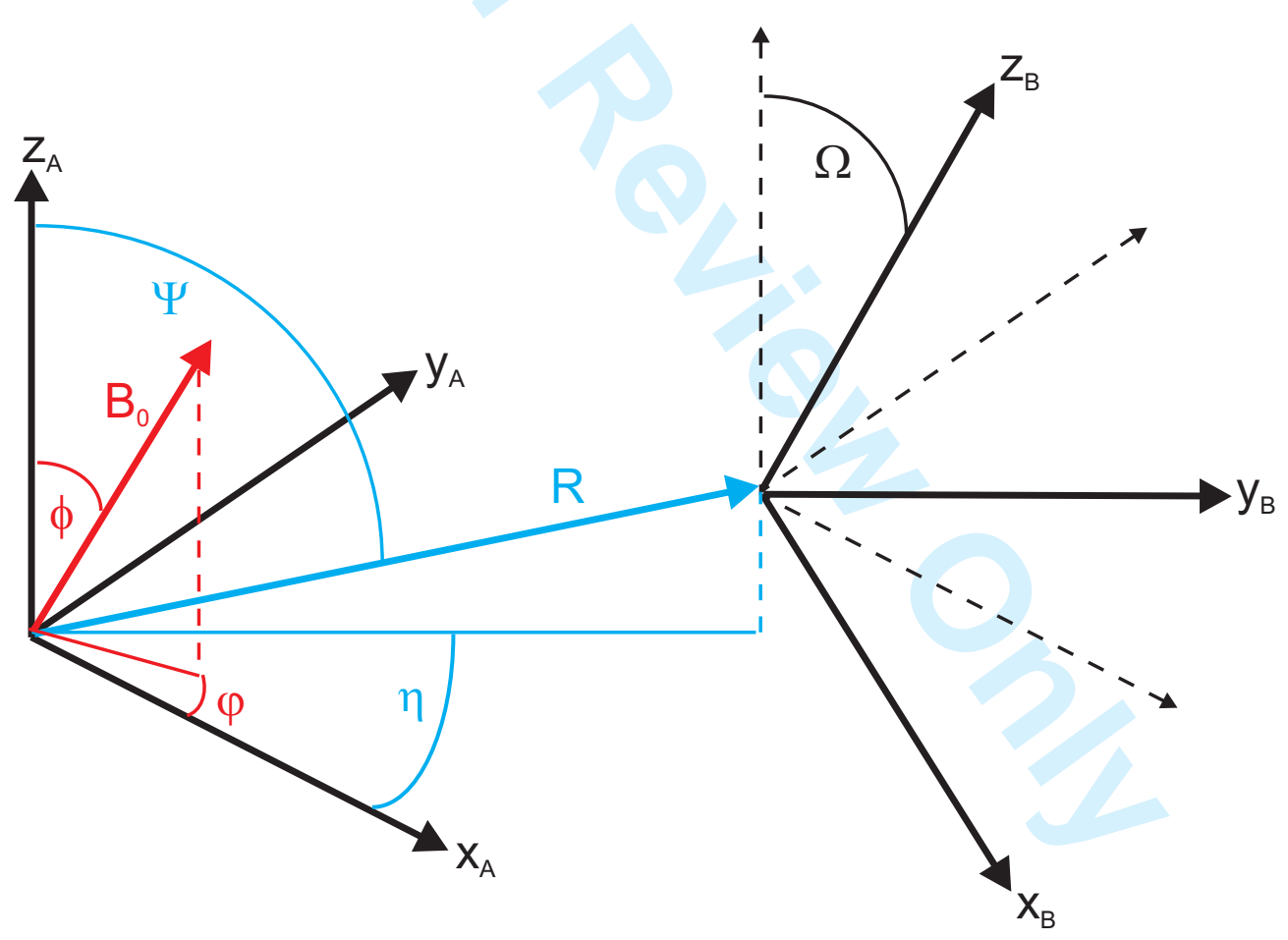

Figure 3: Structures of the two molecules 1 and $\mathbf{2}$ used in the experiments. The geometric model used to create an ensemble of conformers for this biradicals and the molecular axis system are indicated. The nitroxide radicals are assumed to rotate freely around their acetylene bond (cone with angle of $22^{\circ}$ ) and the mobility of the bridge is described by a single bending motion with a Gaussian distributed width $\delta \phi$. 
Figure 4: Experimental PELDOR time traces for offset frequencies $\Delta v_{\mathrm{AB}}$ between 40 and $80 \mathrm{MHz}$ for molecule 1 (a) and 2 (b). All time traces are normalized to 1 for $t=0$ and the intermolecular decay is removed by division by a mono-exponential decay, which is determined by fitting the experimental time traces for long $t$ values. Inset: Field swept EPR spectra of the nitroxide with the position of pump and probe frequencies indicated by arrows.

a)

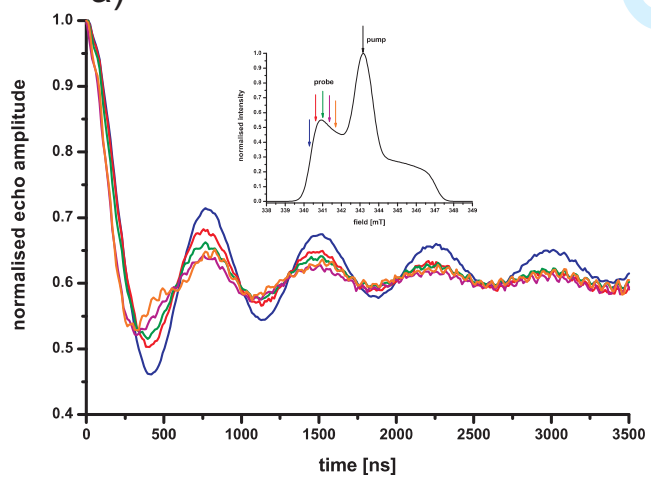

b)

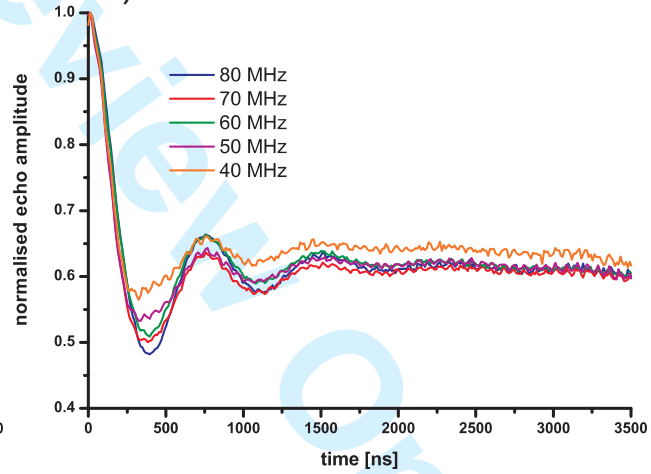

Figure 5: Calculated orientation selection for a nitroxide radical with $\Delta v_{\mathrm{AB}}=80 \mathrm{MHz}$ (left) and $\Delta v_{\mathrm{AB}}=40 \mathrm{MHz}$ (right). A pulse length of $32 \mathrm{~ns}$ and an inhomogeneous linewidth of $6 \mathrm{G}$ is used for the simulations. The colour code gives the intensity of the excitation efficiency (increasing from blue to red). 

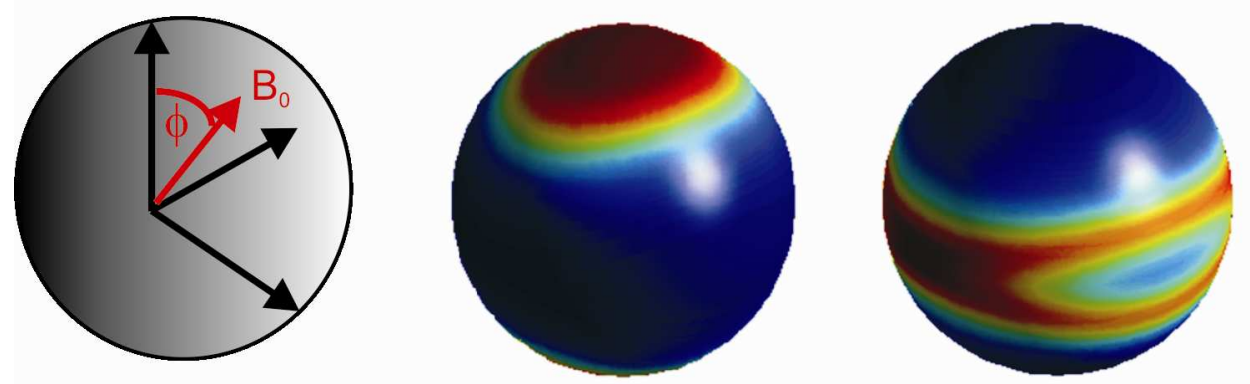

Figure 6: Simulated PELDOR time traces for (deleted: the) $\mathbf{1}$ (a) and $\mathbf{2}$ (b) for $\Delta v_{\mathrm{AB}}=80 \mathrm{MHz}$ (blue, the normalised trace in the graph was shifted by +0.3 in y-direction for reasons of better visibility) and $\Delta v_{\mathrm{AB}}=40$ $\mathrm{MHz}$ (orange). Simulations are shown in black. Parameters for the conformational distribution used for the simulation are given in the text.

a)

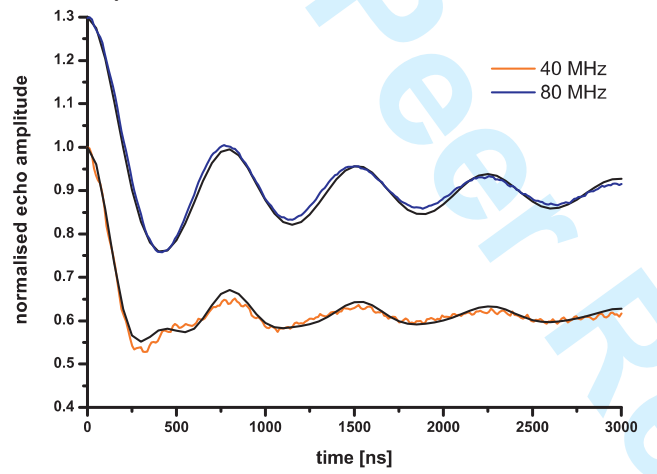

b)

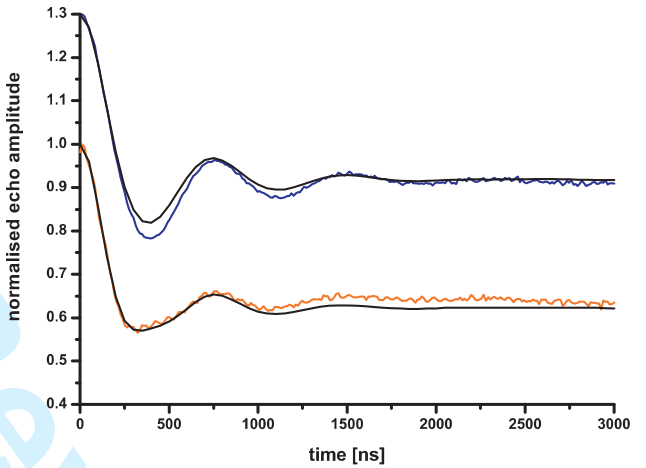

Figure 7: The function $P\left(\Delta v_{\mathrm{AB}}, \theta_{\mathrm{dip}}\right)$ describing the relative intensities of dipolar frequencies contributing to the PELDOR signal of an disordered powder sample for two offset frequencies of $\Delta v_{\mathrm{AB}}=80 \mathrm{MHz}$ (blue) and $\Delta v_{\mathrm{AB}}=$ $40 \mathrm{MHz}$ (orange). For the simulation the geometric model of biradical $\mathbf{1}$ as defined in the text was used. The black line shows the expected $\sin \left(\theta_{\text {dip }}\right)$ intensity for a biradical without any angular correlations, corresponding to a Pake pattern.

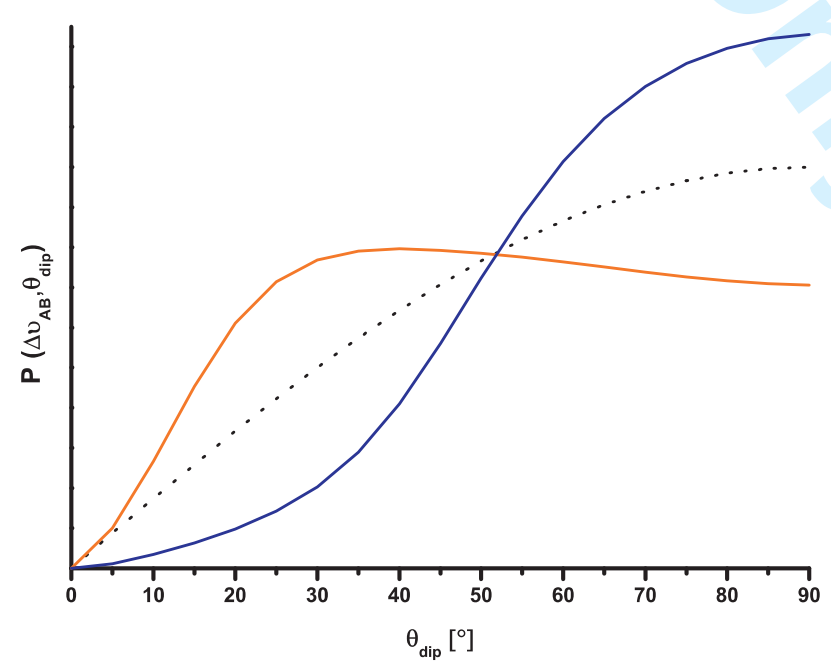




\section{Literature}

[1] a) A.D. Milov, K.M. Salikov, M.D. Shirov. Fiz. Tverd. Tela, 23, 975 (1981). b) A.D. Milov, A.B. Ponomarev, Y.D. Tsvetkov. Chem. Phys. Lett., 110, 67, (1984).

[2] R.G. Larsen, D.J. Singel. J. Chem. Phys., 98, 5134 (1993).

[3] G. Jeschke. Macromol. Rapid Commun., 23, 227 (2002).

[4] O. Schiemann, T.F. Prisner. Quart. Rev. Biophys., in press.

[5] a) R.E. Martin, M. Pannier, F. Diederich, V. Gramlich, M. Hubrich, H.W. Spieß. Angew. Chem. Int. Ed., 37, 2833 (1998). b) M. Pannier, S. Veit, A. Godt, G. Jeschke, H.W. Spieß. J. Magn. Reson., 142, 331 (2000).

[6] A. Weber, O. Schiemann, B. Bode, T.F. Prisner. J. Magn. Reson., 157, 277 (2002).

[7] M. Bennati, T.F. Prisner. Rep. Prog. Phys., 68, 411 (2005).

[8] V.P. Denysenkov, T.F. Prisner, J. Stubbe, M. Bennati. Proc. Natl. Acad. Sci. U. S. A., 103, 13386 (2006).

[9] A. Godt, M. Schulte, H. Zimmermann, G. Jeschke. Angew. Chem. Int. Ed., 45, 7560 (2006).

[10] Y. Polyhach, A. Godt, C. Bauer, G. Jeschke. J. Magn. Reson., 185, 118 (2007).

[11] A. Schweiger, G. Jeschke. Principles of Pulse Electron Paramagnetic Resonance, Oxford University Press, Oxford (2001).

[12] Note that the peak-to-peak distance corresponds to $2 \mathrm{D}_{\text {dip }}$ and the edge-to-edge distance to $4 \mathrm{D}_{\text {dip }}$ in Fourier transformed PELDOR spectra.

[13] A.D. Milov, A.G. Maryasov, Y.D. Tsvetkov. Appl. Magn. Reson., 15, 107 (1998).

[14] a) G. Jeschke, G. Panek, A. Godt, A. Bender, H. Paulsen. Appl. Magn. Reson., 26, 223 (2004). b) Y.-W. Chiang, P.P. Borbat, J.H. Freed. J. Magn. Reson., 172, 279 (2005).

[15] a) B.E. Bode, D. Margraf, J. Plackmeyer, G. Dürner, T.F. Prisner, O. Schiemann. J. Am. Chem. Soc., 129, 6736 (2007). b) O. Schiemann, N. Piton, J. Plackmeyer, B.E. Bode, T.F. Prisner, J.W. Engels. Nat. Protoc., 2, 904 (2007).

[16] G. Jeschke, A. Bender, H. Paulsen, H. Zimmermann, A. Godt. J. Magn. Reson., 169, 1 (2004).

[17] B.L. Farmer, B.R. Chapman, D.S. Dudis, W.W. Adams. Polymer, 34, 1588 (1993). 